\title{
Counterexamples for classical operators on Lorentz-Zygmund spaces
}

by

ROBERT SHARPLEY* (Columbia, S. C.)

Abstract. Lower estimates in terms of Calderón maximal operators $S_{\sigma}$ are provided for various classical operators which appear in Fourier analysis: Hilbert transform, fractional integrals, and convolution. Necessary conditions for the boundedness of the operators $S_{\sigma}$ on Lorentz-Zygmund spaces are produced which complement the sufficient conditions given recently by Bennett and Rudnick. By a combination of these results, pairs of Lorentz-Zygmund spaces (triples in the case of convolution) for which the specified classical operators are bounded are characterized in terms of their indices.

\$ 1. Introduction. A major activity in Fourier Analysis is the investigation of the behavior of operators, intimately related to the Fourier transform, on function spaces. The spaces are usually of the types: restrictions on growth, restrictions on smoothness, or mixtures of these two. In this paper we shall be concerned with the first class of spaces. In particular, we seek to characterize pairs of spaces $(X, Y)$ so that particular operators are bounded from $X$ to $Y$ where the spaces are both drawn from the class of spaces known as Lorentz-Zygmund spaces [1]. This class includes the standard $L^{p}, L^{p, q}$, and $L^{p}(\log L)^{\alpha}$ spaces which historically have been the most widely used to study the relative growth behavior of functions and their transforms. To get sufficient conditions for an operator to be bounded, one typically obtains certain endpoint estimates for the operator and then applies interpolation theory to get intermediate estimates. A particularly powerful tool in interpolation theory is the maximal operator $S_{\sigma}$ of Calderón [6], [22], [1], [7]. In fact, if an operator $T$ satisfies certain weak type endpoint estimates, then

$$
(T f)^{*}(t) \leqslant c S_{\sigma}\left(f^{*}\right)(t) \text { a.e. }
$$

where $f^{*}$ is the decreasing rearrangement of $f$. Normally, $S_{\sigma}$ is easier to investigate than the operators $T$. Once boundedness conditions are obtained for $S_{\sigma}$, (1.1) gives an estimate for $T$. Recently, O. Bennett and K. Rudnick

* This research was supported in part by National Science Foundation Grant No. MCS 77-03666. 
[1] have shown that using $S_{\sigma}$ one obtains not only the standard intermediate estimates, but also the limiting cases "around the two endpoints". The main purpose of this paper is to, first of all, show that for important classical operators (Hilbert transform, fractional integrals, convolution) lower pointwise estimates are obtained in terms of the same operators $S$ (the reverse of inequality (1.1)). Secondly, we show that conditions derived in [1], [22] for $S_{\sigma}$ are best possible. Thus combining these, one can obtain tight estimates for the classical operators on the Lorentz-Zygmund spaces. Finding "optimal pairs" is by no means new, but has been largely overlooked after the fundamental paper of Calderón [6] appeared. It seem that this idea has promise in the more general setting of Banach spaces. Recently, the operators $S_{\sigma}$ have been shown to describe operations in the Banach space setting with many applications to analysis [7]. Hopefully, techniques in this paper can be used to get best possible results in many of these cases as well.

In Section 2 we give necessary conditions of two types: ones giving pointwise lower estimates in terms of Calderón operators, and ones dealing with the fundamental functions of the spaces. Section 3 contains an important embedding result (Proposition (3.1)) which is used to get best possible convolution theorems for Lorentz-Zygmund spaces. We proceed in a similiar way in Section 4 to get "tight" theorems for the Calderón operators. Using the known upper estimates (as in (1.1)) and the lower estimates of Section 2 we can characterize pairs of Lorentz-Zygmund spaces so that certain classical operators are bounded from one space to the other. We shall use the remainder of this section to introduce the pertinent definitions and notation.

We deal with Lebesgue measurable functions both on the circle $\boldsymbol{T}=[0,1)$ with normalized measure and on the line $\boldsymbol{R}=(-\infty, \infty)$. Two functions $f$ and $g$ are called equimeasurable if $m\{s \mid g(s)>t\}$ $=m\{s \mid f(s)>t\}$ for each $t$ where $m$ is the Lebesgue measure. If $f$ is a measurable function which is finite almost everywhere on one of these two measure spaces, then the decreasing rearraingement of $f$ is defined to be the almost everywhere unique positive decreasing function $f^{*}$ on $(0, \infty)$ which is equimeasurable with $|f|$. Notice that when $f$ has support on $\boldsymbol{T}$, then $f^{*}$ has support on $[0,1)$. Also, notice that we use the term decreasing in the wide sense and will continue to do so throughout the paper.

A Banach space $X$ of Lebesgue measurable functions (on either $\boldsymbol{T}$ or $\boldsymbol{R}$ ) is called a rearrangement invariant Banach function space [14:] if the following conditions are satisfied:

(1.2) if $g$ is equimeasurable with some $f \in X$, then $g \in X$ and $\|g\|_{X}=\|f\|_{X}$;

(1.3). $\quad|g| \leqslant|f|$ a.e., $f \in X$ implies $g \in X$ and $\|g\|_{X} \leqslant\|f\|_{X}$;

(1.4) if $m E<\infty$, then there exists a constant $c$ (depending possibly on $E)$ such that for each $f \in X, \int_{E I} f d s \leqslant c\|f\|_{X}$;
(1.5) if $m E<\infty$, then $\left\|\chi_{E}\right\|_{X}<\infty$;

(1.6) if $0 \leqslant f_{n} \uparrow f$ a.e. and $f_{n} \in X$ with $\left\|f_{n}\right\|_{X} \leqslant M<\infty$, then $f \in X$ and $\|f\|_{X} \leqslant M$.

The Lorentz-Zygmund space $L^{p, a}(\log L)^{a}, 0<p, a \leqslant \infty,-\infty<a<\infty$ is defined to be the space of all measurable functions $f$ so that the functional

$$
\|f\|_{p, a ; \alpha}=\left\{\int_{0}^{\infty}\left[f^{*}(t) t^{1 / p}(1+|\ln t|)^{\alpha}\right]^{a} \frac{d t}{t}\right\}^{1 / a}
$$

is finite where the definition is modified in the normal way for $a=\infty$. For $p=\infty, L^{p, a}(\log L)^{\alpha}=\{0\}$ unless either $\alpha+1 / a<0$ or $\alpha=1 / a=0$. For this reason we exclude other cases of $a$ and $\alpha$ when $p=\infty$. For $p>1$ (and some cases of $p=1$ ) the functional given by (1.7) is equivalent to a norm. In all the other cases (1.7) determines a topology on $L^{p, a}(\log L)^{a}$ which can be generated by a complete invariant metric [9] and is therefore an $F$-space. It is not hard to see that in all cases $L^{p, a}(\log L)^{a}$ satisfies (1.2) through (1.6). We still call these rearrangement invariant spaces. For $\alpha=0$ these are the standard Lorentz spaces $L^{p, a}$, while for $1 \leqslant a=p<\infty$ they are the Orlicz spaces $L^{p}(\log L)^{a p}$. For $a=p=\infty$, they produce the exponential classes of Zygmund (see [1] for further details). We note here that for $a, p \geqslant 1$ these are special cases of the so called $A_{\alpha}(X)$ spaces [20].

The Calderón operator $S_{\sigma}$ for an interpolation segment $\sigma=\left(p_{1}, q_{1}\right.$; $\left.p_{2}, q_{2}\right), p_{1}<p_{2}, q_{1} \neq q_{2}$ is defined by

$$
S_{\sigma}(f)(t)=\int_{0}^{\infty} f(s) \min \left(\frac{s^{1 / p_{1}}}{t^{1 / q_{1}}}, \frac{s^{1 / p_{2}}}{t^{1 / \alpha_{2}}}\right) \frac{d s}{s}
$$

for $f$ defined on $(0, \infty)$. Notice that for functions defined on the circle $\boldsymbol{T}$, the integration in (1.8) is only from 0 to 1.

The fundamental function $\varphi_{X}[19],[20]$ of a rearrangement invariant Banach function space $X$ is given by

$$
\varphi_{X}(t)=\left\|\chi_{E}\right\|_{X} \quad \text { where } \quad m E=t .
$$

For the Lorentz-Zygmund spaces $L^{p, a}(\log L)^{a}$, we have

$$
\varphi_{X}(t) \approx t^{1 / p}(1+|\ln t|)^{a}
$$

This equivalence also holds in the case $p=\infty$, when $0<t<1$. We remark that the constant $c$ is used to denote an unspecified but fixed constant when it appears on the main line of an inequality. This constant may change from line to line.

Finally, the author would like to express his thanks to Professor Colin Bennett who explained the work in [1] during private conversations with the author. 
§ 2. Lower estimates for classical operators. In this section we give necessary results for certain classical operators to be bounded on rearrangement invariant spaces. Some of these appear as rearrangement inequalities involving Calderón's maximal operators, while others use the fundamental functions of the spaces involved. Sufficient conditions in terms of the Calderón operators have been given much attention $[6],[5]$, $[20],[11],[22],[24],[1]$, but necessary conditions with a few exceptions $[6],[4],[8]$ have been largely overlooked.

We begin by considering the bilinear operation of convolution from which most of our results follow. The convolution of two measurable functions $f$ and $g$ is defined by

$$
f * g(t)=\int_{-\infty}^{\infty} f(t-s) g(s) d s
$$

when the right hand side is finite a.e. For periodic functions the normalized integral is, of course, taken over $[0,1)$.

LEMara 2.1. If $g$ is a measurable function which is positive and decreasing for $t>0$ and $I_{g}(f)=f * g$ exists (at least in the prinoipal value sense) for each $f$ in some rearrangement invariant space $X$ over $\boldsymbol{R}$, then for each $f \in X$ there is an equimeasurable function $f_{1}$ such that

$$
\left(f_{1} * g\right)^{*}(t) \geqslant g(2 t) \int_{0}^{t} f^{*}(s) d s+\int_{t}^{\infty} f^{*}(s) g(2 s) d s
$$

holds for $t>0$.

Proof. For each $f$, let $f_{1}(t)=f^{*}(-t) \chi_{(-\infty, 0)}(t)$, then for $t>0$

$$
\begin{aligned}
\left(f_{1} * g\right)(t) & =\int_{-\infty}^{\infty} f_{1}(s) g(t-s) d s=\int_{0}^{\infty} f^{*}(s) g(t+s) d s \\
& \geqslant \int_{0}^{\infty} f^{*}(s) g(2 \max (s, t)) d s=g(2 t) \int_{0}^{t} f^{*}(s) d s+\int_{t}^{\infty} f^{*}(s) g(2 s) d s
\end{aligned}
$$

since $g$ decreases and $(s+t) \leqslant 2 \max (s, t)$. But by differentiating the function on the right hand side of (2.1) we see that it decreases for $t>0$. Since $\left(f_{1} * g\right)$ dominates this decreasing function, so does $\left(f_{1} * g\right)^{*}$.

For functions $f$ defined on the circle $\boldsymbol{T}, f^{*}$ has support on $[0,1)$ since we have normalized the measure. Using the same argument as in the previous lemma, we have

LEMna 2.2. Suppose that $X$ is a rearrangement invariant space over $\boldsymbol{T}$, $g$ is a positive decreasing function on $(0,1 / 4)$, and $f * g$ exists in the principal value sense whenever $f$ belongs to $X$. Then for each $f$ in $X$ there exists an $f_{1}$ with $f_{1}^{*} \leqslant f^{*}$ (and hence $\left.f_{1} \in X\right)$ so that

$$
\left(f_{1} * g\right)^{*}(t) \geqslant g(2 t) \int_{0}^{t} f^{*}(s) d s+\int_{t}^{1 / 8} f^{*}(s) g(2 s) d s, \quad 0 \leqslant t \leqslant \frac{1}{8} .
$$

Remark. Actually, for positive symmetrically decreasing functions $f$ and $g$ one can get

$$
(f * g)^{*}(t) \geqslant(\mathbf{1} / 2)\left(g(t) \int_{0}^{t} f(s) d s+\int_{i}^{\infty} f(s) g(s) d s\right) .
$$

In particular, for $t>0$

$$
(f * g)(t) \geqslant \int_{0}^{t} f(t-s) g(s) d s \geqslant \int_{0}^{t} f(t-s) d s g(t)^{*}=\int_{0}^{t} f(u) d \dot{u} g(t) .
$$

Also,

$$
(f * g)(t) \geqslant \int_{t}^{\infty} f(t-s) g(s) d s=\int_{t}^{\infty} f(s-t) g(s) d s \geqslant \int_{t}^{\infty} f(s) g(s) d s
$$

since $f$ decreases and hence $f(s-t) \geqslant f(s)$ when $0<t<s$. Combining the above results and noticing that $g(t) \int_{0}^{t} f(s) d s+\int_{i}^{\infty} f(s) g(s) d s$ decreases, we reach the desired conclusion. Hence, for positive symmetrically decreasing $f$ and $g$ there holds

$$
(f * g)^{*}(t) \geqslant \int_{0}^{t} f(u) d u g(t) \geqslant t f(t) g(t) .
$$

O'Neil observed this in his original paper [16]. This type of condition shows that $(f * g)^{*}(t) \geqslant S_{\sigma}(f, g)(t)$ for the bilinear Calderón. operator [22] corresponding to the segment $\sigma=[(1,1 ; 1),(1, \infty ; \infty),(\infty, 1 ; \infty)]$.

Lemmas 2.1 and 2.2 allows us to get lower estimates for many classical convolution operators. The first such example is given by the Hilbert transform.

$$
H f(t)=\text { p.v. } \frac{1}{\pi} \int_{-\infty}^{\infty} f(s) \frac{d s}{t-s}
$$

and is implicit in [4].

CoRoLr.ARY 2.3. Suppose the domain of $H$ contains $X$, a rearrangement invariant function space on $\boldsymbol{R}$, then for each $f$ in $X$ there is an equimeasurable function $f_{1}$ so that

$$
\left(H f_{1}\right)^{*}(t) \geqslant 1 /(4 \pi) S_{\sigma}\left(f^{*}\right)(t)
$$

where $\sigma=(1,1 ; \infty, \infty)$. In particular, if $H(X) \subseteq Y$ a rearrangement invariant space, then $S_{\sigma}$ is a bounded operator from $X$ to $Y$ : 
The first part follows by applying Lemma 2.1 to the function $g(t)=1 / t$ while the second follows immediately from the closed graph theorem.

Using estimates of $O^{\prime} N e i l$ and Weiss [18], it has been shown [6], [4], [1] for rearrangement invariant spaces that $S_{\sigma}$ being bounded implies the Hilbert transform as well. Using techniques of Boyd and Bennett, similar results are obtained in [8] for the conjugate operator on $\boldsymbol{T}$, where

$$
\tilde{f}(t)=\mathrm{p} \cdot \mathrm{v} \cdot \int_{0}^{1} f(s) \cot (\pi(t-s)) d s .
$$

We have the easy estimate:

COROLLARY 2.4. If the domain of the conjugate operator contains $X$, then for each $f$ in $X$, there is a function $f_{1}$ so that $f_{1}^{*} \leqslant f^{*}, f_{1}$ belongs to $X$, and

$$
\left(\tilde{f}_{1}\right)^{*}(t) \geqslant \frac{1}{2 \sqrt{2} \pi}\left(\frac{1}{t} \int_{0}^{t} f^{*}(s) d s+\int_{t}^{1 / 8} f^{*}(s) \frac{d s}{s}\right), \quad 0<t \leqslant 1 / 8
$$

In particular, if $X$ and $Y$ are rearrangement invariant spaces on $\boldsymbol{T}$ such that $\tilde{X} \subseteq Y$, then it is necessary that the Calderón operator $S_{\sigma}$ for the circle where $\sigma=(1,1 ; \infty, \infty)$ be bounded from $X$ to $Y$.

Proof. Since constants always belong to rearrangement invariant spaces of the circle it is obvious from (2.3) that $S_{\sigma}\left(f^{*}\right) \chi_{(0,1 / 8)}$ bolongs to $Y$ when $\tilde{f}_{1}$ does. This follows since $S_{\sigma}\left(f^{*}\right)$ and the right hand side of $(2.3)$ differ only by the constant $\int_{1 / 8}^{1} f^{*}(s) \frac{d s}{s}$ on $(0,1 / 8)$. But $S_{\sigma}\left(f^{*}\right)$ decreases so $S_{\sigma}\left(f^{*}\right) \chi_{(1 / 8,1)} \leqslant S_{\sigma}\left(f^{*}\right)(1 / 8)$ which with the previous reasoning, implies $S_{\sigma}\left(f^{*}\right)$ belongs to $Y$. By the closed graph theorem $S_{\sigma}$ must in fact be bounded from $X$ to $Y$.

Relation (2.3) is established by applying Lemma 2.2 to $g(t)=\cot (\pi t)$ and then noticing that $\cot (s) \geqslant \frac{\cos (\pi / 4)}{(s)}$ for $0 \leqslant s \leqslant \pi / 4$.

If we consider radially decreasing functions on $\boldsymbol{R}^{n}$, we can show, using the proof of Lemma 2.1 and changing to spherical coordinates, that

$$
(f * g)^{*}(t) \geqslant c_{n} \int_{0}^{\infty} f^{*}(s) g^{*}\left(2^{n} \max (s, t)\right) d s
$$

where $c_{n}$ depends only on the dimension since in this case $f^{*}\left(|s|^{n}\right) \approx f(s)$. If we let $g_{\alpha}(t)=|t|^{a-n}, 0<a<n$, then $f * g_{a}=R_{\alpha}(f)$ are just the Riesz potentials of order $\alpha$ on $\boldsymbol{R}^{n}$. By inequality (2.4) we have for decreasing positive $f$ on $\boldsymbol{R}^{n}$ that

$$
R_{a}(f)(t) \geqslant a S_{\alpha}(f)(t)
$$

where $\sigma=\left(1, \frac{n}{n-a} ; n / a, \infty\right)$ since $g^{*}(t) \approx t^{\alpha / n-1}$. Combining this with the well-known sufficient conditions [6], we have that a necessary and sufficient condition that $R_{a}$ is bounded from $X\left(\boldsymbol{R}^{n}\right)$ to $Y\left(\boldsymbol{R}^{n}\right)$ (where $X\left(\boldsymbol{R}^{n}\right)$ is the set of measurable functions $f$ on $\boldsymbol{R}^{n}$ with norm $\left.\left\|f^{*}\right\|_{X}\right)$ is that $S_{\sigma}$ is bounded from $X$ to $Y$.

The fractional integral of order $\alpha$ for periodic functions $f$ with mean value zero is given by

$$
f_{a}(t)=\int_{0}^{1} f(s) \Psi_{a}(2 \pi(t-s)) d s
$$

where

$$
\begin{aligned}
\Gamma(\alpha) \Psi_{\alpha}(t) & =\left(t^{\alpha-1}+\lim _{n \rightarrow \infty}\left\{\sum_{k=1}^{n}(t+2 \pi k)^{\alpha-1}-\frac{(2 \pi)^{\alpha-1} n^{\alpha}}{\alpha}\right\}\right) \\
& =t^{\alpha-1}+\Gamma(\alpha) r_{\alpha}(t) / 2 \pi, \quad 0<\alpha<1
\end{aligned}
$$

(see [25], p. 135, vol. II). But the function $r_{a}$ is infinitely differentiable on $[0,2 \pi]$ so for functions $f$ with mean value zero the statements (i) $f_{\alpha} \in \mathbf{Y}$ for each $f \in X$ and (ii) $f * t^{\alpha-1} \in Y$ for each $f \in X$ are equivalent. By subtracting $\int_{0}^{1} f(t) d t$ from $f$ and noticing that constants always belong to $X$ we see that (i) and (ii) are equivalent for all $f$. But now by Lemma 2.2 there holds

COROLLARY 2.5. A necessary condition that the fractional integral $f_{\alpha} \in Y$ for each $f$ in $X$, where $X$ and $Y$ are rearrangement invariant spaces, is that $S_{\sigma}$ is a bounded operator from $X$ to $Y$ for $\sigma=(1,1 / 1-\alpha ; 1 / \alpha, \infty)$.

It is well known [6], [17], [15], [1], [24] that the condition is also sufficient and, in fact,

$$
\left(f_{a}\right)^{*}(t) \leqslant S_{a}\left(f^{*}\right)(t), \quad 0 \leqslant t \leqslant 1 .
$$

Our last example of lower estimates is for the real Laplace transform

$$
L(f)(t)=\int_{0}^{\infty} f(s) e^{-s t} d s .
$$

For positive decreasing $f$, there holds

$$
L(f)(t) \geqslant \int_{0}^{1 / t} f(s) e^{-s t} d s \geqslant e^{-1} \int_{0}^{1 / t} f(s) d s=e^{-1} \int_{0}^{\infty} f(s) d \min \left(s^{1} / t^{0}, s^{0} / t^{1}\right) .
$$

Since $L$ is of weak types $(1, \infty)$ and $(\infty, 1)$ (see [6]), then this inequality. can be inverted with a change of constant if we replace $f$ with $f^{*}$. 
In [20], Theorem 5.7, it is shown that a necessary condition that $S_{\sigma}(X) \subseteq Y$ is that for all $s$ and $t$

$$
c \varphi_{X}(s) \geqslant \varphi_{Y}(t) \min \left(\frac{s^{1 / p_{1}}}{t^{1 / \alpha_{1}}}, \frac{s^{1 / p_{2}}}{t^{1 / \alpha_{2}}}\right)
$$

where $\sigma=\left(p_{1}, q_{1} ; p_{2}, q_{2}\right)$. This results from the fact that

$$
c\left\|\chi_{(0, s)}\right\|_{X} \geqslant\left\|S_{\sigma}\left(\chi_{(0, s)}\right)\right\|_{Y} \geqslant \varphi_{Y}(t) S_{\sigma}\left(\chi_{(0, s)}\right)(t)
$$

must hold, which implies (2.5) upon evaluation of $S_{\sigma}\left(\chi_{(0, s)}\right)(t)$. Letting $m=\left(1 / q_{1}-1 / q_{2}\right) /\left(1 / p_{1}-1 / p_{2}\right)$ and $b=1 / q_{1}-m / p_{1}$ (i.e. the slope and $y$ intercept of the segment $\sigma$ ), then we see that it is necessary that

$$
\varphi_{Y}(t) \leqslant c \varphi_{X}\left(t^{m}\right) \cdot t^{b} \quad \text { for all } t
$$

if $S_{\sigma}(X) \subseteq Y$, by letting $s=t^{m}$ in (2.5). Condition (2.6) along with other conditions can be shown to be sufficient in certain cases for boundedness of $S_{\sigma}$ [11], [23], [24]

We end this section with a necessary condition on spaces $X$ and $Y$ for which $\hat{X} \subseteq Y$. Here we use $\hat{f}$ to denote the Fourier transform of $f$ on the line given by

$$
\hat{f}(t)=(2 \pi)^{-1 / 2} \int_{-\infty}^{\infty} f(s) e^{-i s t} d s .
$$

A similiar result holds for the Fourier coefficients of a function on the circle.

THEOREM 2.6. If $\hat{X} \subseteq Y$ for rearrangement invariant spaces $X$ and $Y$ on $\boldsymbol{R}$, then

$$
t \varphi_{Y}(1 / t) \leqslant c \varphi_{X}(t), \quad t>0 .
$$

Proof. An easy calculation gives

$$
\hat{\chi}_{(-t, t)}(s)=2(2 \pi)^{-1 / 2} t(\sin (s t) /(s t))
$$

so that

(2.7)

$$
\left(\hat{\chi}_{(-t, t)}\right)^{*}(s)=2(2 \pi)^{-1 / 2} t g^{*}(s t)
$$

where $g(s)=|\sin (s) / s|$. But

$$
g(s) \geqslant(2 \pi)^{-1 / 2} \sum_{-\infty}^{\infty}|s|^{-1} \chi_{E_{k}}(s)
$$

where $E_{k}=[\pi / 6+k \pi, 5 \pi / 6+k \pi]$, so

$$
g^{*}(s) \geqslant \frac{1}{2}(2 \pi)^{-1 / 2} \sum_{0}^{\infty} \frac{1}{(k+1) \pi} \chi_{[2 \pi k / 3,2 \pi(k+1) / 3)}(s) \geqslant(2 \pi)^{-3 / 2} \min (1,1 / s) .
$$

If $\hat{X} \subseteq Y$, then there must in fact hold

$$
\|\hat{f}\|_{Y} \leqslant c\|f\|_{X} .
$$

Applying this to $f(s)=\chi_{(-t, t)}(s)$, we see by (2.7) and (2.8) that $t \varphi_{Y}(1 / t) \leqslant t \sup _{s}\left\{\min (1,1 / t s) \varphi_{Y}(s)\right\} \leqslant c \sup _{s}\left(\hat{\chi}_{(-t, t)}\right)^{*}(s) \varphi_{Y}(s) \leqslant c\left\|\hat{\chi}_{(-t, t)}\right\|_{Y}$

$$
\leqslant o \varphi_{X}(2 t) \text {. }
$$

But $p_{X}(2 t) \leqslant 2 p_{X}(t)$ for all $t$ establishes the result.

Jodeit and Torchinsky [1.0] have shown that a similar condition, namely

$$
s \varphi_{B}(1 / s t) \leqslant c \varphi_{A}(s / t), \quad 0<t \leqslant 1, s>0
$$

is necessary and sufficient for the Fourier transform to be bounded from the Orlicz space $L_{A}$ to $L_{B}$.

\$ 3. Embeddings and convolution. In this section we consider LorentzZygmund spaces on the circle with normalized measure. In order to facilitate the statement and proofs of most of the results in this section and the next, we distinguish certain subsets of the closed upper half plane $\boldsymbol{R}_{+}^{2}$ $=\{(\alpha, 1 / a) \mid-\infty<a<\infty, 0<a \leqslant \infty\}$. Let

(3.1) $\quad \boldsymbol{I}(\beta, 1 / b)=\{(\alpha, 1 / a) \mid \alpha+1 / a<\beta+1 / b, 0<a<b\} \cup$

$$
\cup\{(\alpha, 1 / a) \mid \alpha \leqslant \beta, b \leqslant a \leqslant \infty\}
$$

and

$$
\boldsymbol{E}(\beta, 1 / b)=\bigcup_{\substack{\alpha+1 / a=\beta+1 / b \\ b \leqslant a \leqslant \infty}} \boldsymbol{I}(\alpha, 1 / a)
$$
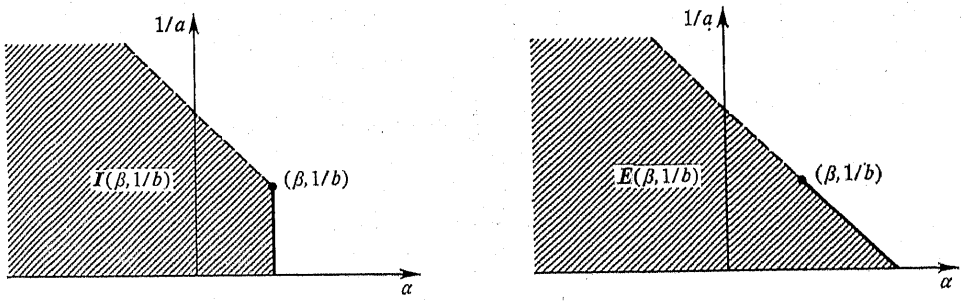

Fig. 1

Using the results of [1], there holds

Proposrtron 3.1. A necessary and sufficient condition that 
is that either (i) $r<q$, or

$$
\text { (ii) } r=q \text { and }(\gamma, 1 / e) \in\left\{\begin{array}{lll}
\boldsymbol{I}(\beta, 1 / b) & \text { when } & q<\infty, \\
\boldsymbol{E}(\beta, 1 / b) & \text { when } & q=\infty .
\end{array}\right.
$$

Proof. By the closed graph theorem the containment (3.3) is actually a continuous embedding. The sufficiency is Theorems 9.1, 9.3, and 9.5 of [1]. For the necessity we first notice that $r>q$ implies that the positive decreasing function $f(t)=t^{-(1 / r+1 / q) / 2}$ belongs to $L^{a, b}(\log L)^{\beta}$ but not to $L^{r, c}(\log L)^{\gamma}$. Hence we can assume $q=r$. We also assume that $q<\infty$ leaving the case $q=\infty$ to the reader as the proofs are similar but somewhat simpler. If, in this case, $(\gamma, 1 / e) \notin \boldsymbol{I}(\beta, 1 / b)$, then either $(\gamma>\beta$, $b \leqslant c \leqslant \infty)$ or $(\gamma+1 / c \geqslant \beta+1 / b, 0<c<b)$. In the first situation, the continuous inclusion (3.3) implies

$$
o \geqslant \sup _{0<t<1} \frac{\left\|\chi_{(0, t)}\right\|_{q, c, \gamma}}{\left\|\chi_{(0, t)}\right\|_{q, b, \beta}} \approx \sup _{0<t<1} \frac{(1-\ln t)^{\gamma}}{(1-\ln t)^{\beta}}
$$

which leads to a contradiction when $\gamma>\beta$. In the second case we first notice that we might as well assume $\gamma+1 / c=\beta+1 / b, 0<0<b$ since the sufficiency of the theorem applied to $\gamma^{\prime}=\beta+1 / b-1 / o$ yields $L^{\alpha, c}(\log L)^{\gamma}$ $\subseteq L^{a, c}(\log L)^{\gamma^{\prime}}$. We consider the function $f$ (depending on $q, \beta, b, \delta$ ) given by

$$
f(t)=\left(|t|^{1 / \alpha}(1-\ln |t|)^{\beta+1 / b}[\ln (1-\ln |t|)]^{\delta}\right)^{-1} \chi_{(0, u)}(|t|)
$$

where $u$ is chosen appropriately small so that $f$ decreases on $(0, u)$. Letting $\delta=1 / e$ we see by a direct calculation that $f$ belongs to $L^{q, b}(\log L)^{\beta}$ but not to $L^{q, c}(\log L)^{\gamma}$.

Remark 3.2. In the first case of the necessity of this theorem we could construct $f$ explicitly by the formula

$$
f(t)=\sum a_{n} \chi_{\left(0, t_{n}\right)}
$$

where $\quad a_{n}=\left(\left\|\chi_{\left(0, t_{n}\right)}\right\|_{a, b ; \beta} n^{2 d}\right)^{-1}, \quad t_{n}=\exp \left(-n^{3 a /(\gamma-\beta)}\right), \quad$ and $\quad d=(b+1) / b$ rather than rely upon the closed graph theorem for $T$-spaces.

We should also mention that certain cases of the necessity were also mentioned in [1], Remark 9.4. There they show that inclusion (3.3) does not hold when either $(b=1, \beta=0, a=\infty, \gamma=\varepsilon)$ or $(b=\infty$, $\beta=1, o=1, \gamma=0)$. We have merely tightened up these in order to get necessary and sufficient conditions for the classical operators in Section 2 to be bounded on the Lorentz-Zygmund spaces. Our first indication of this is to completely describe intermediate convolution results for these spaces on $\boldsymbol{T}$
Trmorram 3.3. Suppose $1<p, q, r<\infty$. A necessary condition that is that

$$
L^{p, a}(\log L)^{\alpha} * L^{\alpha, b}(\log L)^{\beta} \subseteq L^{r, c}(\log L)^{\gamma}
$$

hold. If strict inequality holds in (3.7), then the convolution theorem (3.6) holds for all ehoices of $\alpha, \alpha, \beta, b, \gamma, o$. If equality holds in the relation (3.7), then a necessary and sufficient condition for (3.6) is that $(\gamma, 1 / c) \in \boldsymbol{I}(\alpha+\beta$, $1 / a+1 / b)$.

Proof. To show (3.7) is necessary we first show that (3.6) is equivalent to the continuity of convolution, i.e.

$$
\|f * g\|_{r, c ; \gamma} \leqslant o\|f\|_{p, a ; a}\|g\|_{q, b ; \beta} .
$$

If (3.8) holds for no finite $c$, then we can find a sequence of functions $\left\{f_{n}\right\}$ and $\left\{g_{n}\right\}$ such that $\left\|f_{n}\right\|_{p, a ; \alpha}=\left\|g_{n}\right\|_{a, b ; \beta}=1$ but $\left\|f_{n} * g_{n}\right\|_{r, c ; \gamma}>n^{5+2\left(\frac{a+b}{a b}\right)}$. Since $\left|f_{n}\right| *\left|g_{n}\right| \geqslant\left|f_{n} * g_{n}\right|$, and all spaces are rearrangement invariant, we can assume $f_{n}$ and $g_{n}$ are positive. Let

$$
f=\sum n^{-2(1+1 / a)} f_{n}, \quad g=\sum n^{-2(1+1 / b)} g_{n} .
$$

Then $f \in L^{p, a}(\log L)^{a}$ and $g \in L^{\alpha, b}(\log L)^{\beta}$, and so by (3.6) $f * g \in L^{r, c}(\log L)^{\gamma}$. But since convolution is a positive bilinear form,

$$
\|f * g\|_{r, c ; \gamma} \geqslant n^{-4-2\left(\frac{a+b}{a b}\right)}\left\|f_{n} * g_{n}\right\|_{r, c ; \gamma}>n, \quad \text { for each } n .
$$

Hence (3.8) must hold. It is obvious that (3.8) implies (3.6).

Noticing $\chi_{(0, t)} * \chi_{(0, t)}(s) \geqslant s \chi_{(0, t)}(s)$, we see that $\left(\chi_{(0, t)} * \chi_{(0, t)}\right)^{*}(s) \geqslant(t / 2) \chi_{(0, t / 2)}(s)$ and so by substitution into (3.8) we have

$$
\begin{aligned}
(t / 2)(t / 2)^{1 / r}(1-\ln t)^{\gamma} & \leqslant\left\|\chi_{(0, t)} * \chi_{(0, t)}\right\|_{r, c ; \gamma} \\
& \leqslant c t^{1 / p}(1-\ln t)^{\alpha} t^{1 / q}(1-\ln t)^{\beta}
\end{aligned}
$$

or

$$
t^{1+1 / r}(1-\ln t)^{\gamma} \leqslant 0 t^{1 / p+1 / \alpha}(1-\ln t)^{\alpha+\beta}, \quad 0<t \leqslant 1 .
$$

Hence it is necessary that condition (3.7) hold. If $1 / p+1 / q<1 / r+1$, then we choose $1<p_{1}<p, 1<q_{1}<q, r<r_{1}<\infty$ so that $1 / p_{1}+1 / q_{1}$ $=1 / r_{1}+1$. But then by (i) of Proposition 3.1 and by Young's theorem, we get

$$
L^{p, a}(\log L)^{\alpha} * L^{\alpha, b}(\log L)^{\beta} \subseteq L^{p_{1}} * L^{q_{1}} \subseteq L^{r_{1}} \subseteq L^{r, c}(\log L)^{\gamma}
$$

for each selection of $a, \alpha, b, \beta, c, \gamma$. The only remaining case is $1 / p+1 / q$ $=1 / r+1$. By Theorem (4.1) of [15] with $\varphi_{1}(t)=\left\|\chi_{(0, t)}\right\|_{p, a ; a}, \varphi_{2}(t)$ 
$=\left\|\chi_{(0, t)}\right\|_{\alpha, b ; \beta}$, and $\varphi_{3}(t)=\left\|\chi_{(0, t)}\right\|_{r, c ; \gamma}$ we have that (3.6) holds with $\gamma$ $=\alpha+\beta$ and $1 / c=1 / a+1 / b$. The remainder of the sufficiency follows by the embedding in Proposition 3.1. For the necessity we see from (3.9) that $\gamma \leqslant \alpha+\beta$ must hold. Hence to show that $(\gamma, 1 / c) \in \boldsymbol{I}(\alpha+\beta, 1 / a+1 / b)$, we only need to show that

$$
\gamma+1 / c<\alpha+\beta+1 / a+1 / b \text { when } 1 / c>1 / a+1 / b .
$$

Using the formula (3.5), define $f$ with parameters $p, \alpha, a, 1 / a+\varepsilon$ and $g$ with parameters $q, \beta, b, 1 / b+\varepsilon$ where $\varepsilon=(1 / e-(1 / a+1 / b)) / 2$. Since both functions are positive and symmetrically decreasing, a straight forward calculation shows $f \in L^{p, a}(\log L)^{a}$ and $g \in L^{q, b}(\log L)^{\beta}$. By the remark following Lemma 2.2

$$
(f * g)^{*}(t) \geqslant t f(t) g(t)=t^{-1 / r}(1-\ln t)^{-(\alpha+1 / a+\beta+1 / b)}(\ln (1-\ln t))^{-1 / c}
$$

for $t$ appropriately small. The only way $f * g$ could belong to $L^{r, c}(\log L)^{\gamma}$ is for (3.10) to hold when $1 / c>1 / a+1 / b$.

§ 4. Calderón operators. Our aim in this section is to characterize the Lorentz-Zygmund spaces on $[0,1]$, so that for a given interpolation segment $\sigma=\left(p_{1}, q_{1} ; p_{2}, q_{2}\right)$ with $p_{1}<p_{2}$

$$
\text { (4.1) } \quad S_{\sigma}\left(L^{p, a}(\log L)^{\alpha}\right) \subseteq L^{a, c}(\log L)^{\gamma}
$$

keeping in mind the results of $\S 2$. For $\sigma=(1,1 ; \infty, \infty)$ this will characterize the pairs of spaces of this type so that $\tilde{X} \subseteq Y$; for $\sigma=\left(1,(1-\theta)^{-1}\right.$; $\left.\theta^{-1}, \infty\right), \bar{X}_{\theta} \subseteq Y$ is determined where $f_{\theta}$ is the $\theta$ th fractional integral of $f$ on $[0,2 \pi)$ with normalized measure. For the Riesz potentials and Riesz transforms on $\boldsymbol{R}^{n}$ we have similar results but must instead deal with sums and intersections. of Lorentz-Zygmund spaces. We leave these to the interested reader to formulate [1]. We will assume that $q_{1}<q_{2}$ since interpolation segments with positive slopes are the only interesting ones for domain and range spaces both over finite measure spaces.

We first restrict ourselves to only the interesting cases. We observe that it is necessary that $L^{p, a}(\log L)^{a} \subseteq L^{p_{1}, 1}(\log L)^{0}$ in order that $S_{\sigma}(f)$ exist for each $f \in L^{p, a}(\log L)^{a}$. This is due to the fact that $S_{\sigma}\left(f^{*}\right)$ is positive decreasing and $S_{\sigma}\left(f^{*}\right)(1)=\|f\|_{p_{1}, 1 ; 0}$. By the embedding result Proposition 3.1, then either $p_{1}<p$ or $p=p_{1}$ and $(0,1) \in \boldsymbol{I}(\alpha, 1 / a)$. We define

$$
\boldsymbol{R}(\gamma, 1 / c)=\{(\alpha, 1 / a) \mid(\gamma, 1 / c) \in \boldsymbol{I}(\alpha, 1 / a)\} .
$$

The case $p>p_{2}$ is also uninteresting since $\chi_{(0, r)}$ belongs to $L^{p, a}(\log L)^{a}$ but $S_{\sigma}\left(\chi_{(0, r)}\right)$ belongs to no "smaller" space than $L^{\alpha_{2}, \infty}(\log L)^{0}$. To see this, we notice by the embedding Proposition 3.1 that it is enough to consider $L^{a_{2}, c}(\log L)^{\gamma}, \gamma+1 / 0=0$. Here

$$
S_{\sigma}\left(\chi_{(0, r)}\right)(t) \geqslant \frac{r^{1 / p_{2}}}{t^{1 / \alpha_{2}}}, \quad 0<t \leqslant r^{1 / m}
$$

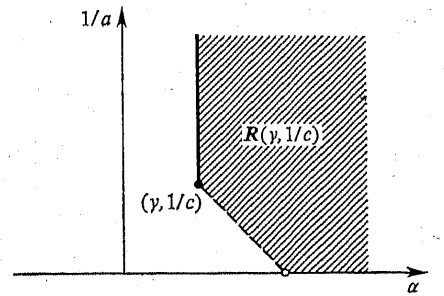

Fig. 2

$$
\left\|S_{\sigma}\left(\chi_{(0, r)}\right)\right\|_{\alpha_{2}, c ; \gamma} \geqslant r^{1 / p_{2}}\left\{\int_{0}^{r / m}(1-\ln t)^{\gamma c} \frac{d t}{t}\right\}^{1 / c}=\infty
$$

since $\gamma c=-1$. So the best that can be said for $p>p_{2}$ is that

$$
L^{p, a}(\log L)^{\alpha} \subset L^{p_{2}, \mathrm{1}}(\log L)^{0} \stackrel{\mathrm{s}_{\sigma}}{\rightarrow} L^{q_{2}, \infty}(\log L)^{0},
$$

for each $0<a \leqslant \infty,-\infty<a<\infty$. We, consequently, need only consider either

$$
p_{1}<p \leqslant p_{2}
$$

$$
(a, 1 / a) \in \boldsymbol{R}(0,1) \text { when } p=p_{1} .
$$

With the domain restricted in this manner, we notice by relation (2.6) that

$$
m / p+b \leqslant 1 / q
$$

where we remind that $m=\left(1 / q_{1}-1 / q_{2}\right) /\left(1 / p_{1}-1 / p_{2}\right)$ and $b=1 / q_{1}-m / p_{1}$. If there is strict inequality in (4.4), then we pick $p_{1}<\bar{p}<p$ and $q<\bar{q}<q_{2}$ so that $m / \bar{p}+b=1 / \bar{q}$. Oondition (4.1) is satisfied for all choices of $a, a, \gamma, c$ since

$$
L^{p, a}(\log L)^{a} \hookrightarrow \rightarrow L^{\bar{p}, 1} \stackrel{\boldsymbol{S}_{\sigma}}{\rightarrow} L^{\bar{\alpha}, 1} \subset \rightarrow L^{q, o}(\log L)^{\gamma}
$$

holds by two applications of Proposition 3.1 and the original weak type theorem of Oalderón [6]. We have now reduced consideration to conditions (4.2) or (4.3) for the domain and

(4.5)

$$
1 / q=m / p+b
$$

for the range.

THEOREM 4.1 (Intermediate case). Suppose $p_{1}<p<p_{2}$ and (4.5) holds, then a necessary and sufficient condition that relation (4.1) is satisfied is that $(\gamma, 1 / c) \in I(a, 1 / a)$. 
Proof. To see that the condition is sufficient, one applies Theorem 5.6 of [20] or Theorem $\mathrm{B}$ of [1] to get

$$
S_{\sigma}\left(L^{p, a}(\log L)^{\alpha}\right) \subseteq L^{a, a}(\log L)^{\alpha} .
$$

Proposition 3.1 provides that (4.1) is true when $(\gamma, 1 / \sigma) \in \boldsymbol{I}(\alpha, 1 / \alpha)$.

On the other hand, if (4.1) holds, then noticing

$$
t^{-b} f^{*}\left(t^{m}\right) \leqslant t^{-1 / q_{1}} \int_{0}^{t^{m}} f^{*}(s) s^{1 / p_{1}} \frac{d s}{s} \leqslant S_{\sigma}\left(f^{*}\right)(t)
$$

we have by a change of variable $u=t^{m}$

$$
\|f\|_{p, c ; \gamma} \leqslant c\left\{\int_{0}^{1}\left[t^{-b} f^{*}\left(t^{m}\right) t^{1 / \alpha}(1-\ln t)^{\nu}\right]^{c} \frac{d t}{t}\right\}^{1 / c} \leqslant o\left\|S_{a} f^{*}\right\|_{\alpha, c ; \gamma} \leqslant c\|f\|_{p, a ; \alpha} .
$$

This just says $L^{p, a}(\log L)^{a} \subseteq L^{p, c}(\log L)^{\gamma}$ and so by Proposition $3.1(\gamma, 1 / 0)$ $\in \boldsymbol{I}(\alpha, 1 / a)$.

Next we obtain the corresponding results in the more delicate endpoint cases. For this we need to partition $\boldsymbol{R}_{+}^{2}$ into sets $\boldsymbol{R}_{1}, \ldots, \boldsymbol{R}_{6}$ given

$$
\begin{aligned}
& \boldsymbol{R}_{1}=\{(0,1 / a) \mid 0<a \leqslant 1\}, \\
& \boldsymbol{R}_{2}=\{(\alpha, 1 / a) \mid 0<a<1,0<a\}, \\
& \boldsymbol{R}_{3}=\{(\alpha, 1 / a) \mid 1 \leqslant a \leqslant \infty, a+1 / a>1\}, \\
& \boldsymbol{R}_{4}=\{(\alpha, 1 / a) \mid 1<a \leqslant \infty, a+1 / a=1\}, \\
& \boldsymbol{R}_{5}=\{(\alpha, 1 / a) \mid 1 \leqslant a \leqslant \infty, a+1 / a<1\}, \\
& \boldsymbol{R}_{6}=\{(\alpha, 1 / a) \mid 0<a<1, \alpha<0\} .
\end{aligned}
$$

These sets are all disjoint and have the property that $\bigcup_{j=1}^{6} \boldsymbol{R}_{j}=\boldsymbol{R}_{+}^{2}$ and $\bigcup_{j=1}^{3} \boldsymbol{R}_{j}=\boldsymbol{R}(0,1)$. Keeping the reductions made earlier in this section in mind, we state

Theorem 4.2 (Right Endpoint Theorem). Suppose $p=p_{1}$, then it is necessary that $(\alpha, 1 / a) \in \boldsymbol{R}(0,1)$. If $q=q_{1}$ (see relation (4.5)), then in order for $S_{\sigma}$ to satisfy relation (4.1) when $(\alpha, 1 / \alpha)$ belongs to the region

(a) $\boldsymbol{R}_{1}$, it is necessary and sufficient that $(\gamma, \mathbf{1} / 0) \in \boldsymbol{E}(0,1 / \infty)$;

(b) $\boldsymbol{R}_{2}$, it is necessary that $(\gamma, 1 / c)$ belong to the closure of $\boldsymbol{E}(\alpha-1 / a, 1 / a)$ and sufficient that $(\gamma, 1 / c) \in \boldsymbol{E}(a-1 / a, 1 / a)$;

(c) $\boldsymbol{R}_{3}$, it is necessary and suffioient that $(\gamma, 1 / c) \in \boldsymbol{E}(\alpha-1,1 / a)$.

THEOREM 4.3 (Left Endpoint Theorem). Suppose $p=p_{2}$ and so by relation (4.5) $q=q_{2}$. In order for $S_{\sigma}$ to satisfy relation (4.1) when $(a, 1 / a)$
belongs to the region (a) $\boldsymbol{R}(0,1)$, it is necessary and sufficient that $(\gamma, 1 / 0) \in \boldsymbol{E}(0,1 / \infty)$;

(b) $\boldsymbol{R}_{4}$, it is necessary and sufficient that $(\gamma, 1 / c)$ betong to the interior of $\boldsymbol{E}(0,1 / \infty)$

(c) $\boldsymbol{R}_{5}$, it is necessary and sufficient that $(\gamma, 1 / 0) \in \boldsymbol{E}(\alpha-1,1 / a)$;

(d) $\boldsymbol{R}_{6}$, it is necessary that $(\gamma, 1 / c)$ belong to the elosure of $\boldsymbol{E}(\alpha-1 / a$, $1 / a)$ and sufficient that $(\gamma, 1 / \theta) \in \boldsymbol{E}(\alpha-1 / a, 1 / a)$.

Remark 4.4. If not for the cases (b) of Theorem 4.2 and (d) of Theorem 4.3 we would have an complete characterization of fractional integration, conjugation, and weak type interpolation for the Lorentz-Zygmund spaces. The most important cases in applications, Theorem 4.2 (c) and Theorem 4.3 (c), are characterized, however. The sufficient conditions of these cases are contained in [1].

By sketching the regions in the completed cases of Theorems 4.2 and 4.3 and investigating the configuration of the sets $\boldsymbol{E}$ as you approach boundaries of the various regions, one is inclined to conjecture, say for example in Theorem $4.2(\mathrm{~b})$, that (4.1) does not hold in the remaining case of $\gamma+1 / e=a, 0<c<a$. But a careful examination of the relative behaviour of $f$ and $S_{\sigma}(f)$ for various functions (powers, logs, etc.) forces us instead to state.

CONJEOIURA. Fror $(\alpha, 1 / a) \in \boldsymbol{R}_{2}$, a necessary and sufficient condition that (4.1) holds with $p=p_{1}, q=q_{1}$ is that $(\gamma, 1 / c)$ belong to the closure of $\boldsymbol{E}(\alpha-1 / \alpha, 1 / a)$. that

Proof of Theorem 4.2. (a) By the definition of $S_{\sigma}$ one easily sees

$$
t^{1 / q_{1}} S_{\sigma}\left(f^{*}\right)(t) \leqslant t^{1 / q_{1}} \int_{0}^{1} f^{*}(s) \min \left(\frac{s^{1 / p_{1}}}{t^{1 / q_{1}}}, \frac{s^{1 / p_{2}}}{t^{1 / q_{2}}}\right) \frac{d s}{s} \leqslant \int_{0}^{1} f^{*}(s) s^{1 / p_{1}} \frac{d s}{s}
$$

or $\dot{S}_{\sigma}\left(L^{p_{1}, 1}(\log L)^{0}\right) \subseteq L^{q_{1}, \infty}(\log L)^{0}$. By the embedding Proposition 3.1 the sufficiency of (a) for (4.1) to hold follows.

To prove the necessity we first notice that it suffices to show that there exists $f \in L^{p_{1}, a}(\log L)^{\alpha}$ such that $S_{\sigma}(f) \notin L^{q_{1}, c}(\log L)^{\gamma}$ when $\gamma+1 / c=0$ and $0<0<\infty$. This is due to the fact that the complement of $\boldsymbol{E}(0,1 / \infty)$ satisfies

$$
\boldsymbol{R}_{-\uparrow}^{2} \backslash \boldsymbol{E}(0,1 / \infty)=\bigcup_{\substack{\gamma+1 / 1=0=0 \\ 0<c<\infty}} \boldsymbol{R}(\gamma, 1 / c)
$$

For if $\left(\gamma^{\prime}, 1 / o^{\prime}\right) \notin \mathbb{E}(0,1 / \infty)$, then there is $(\gamma, 1 / c)$ such that $\left(\gamma^{\prime}, 1 / c^{\prime}\right)$ $\in \boldsymbol{R}(\gamma, 1 / 0)\left(\right.$ i.e. $\left.(\gamma, 1 / o) \in \boldsymbol{I}\left(\gamma^{\prime}, 1 / o^{\prime}\right)\right)$ and $\gamma+1 / 0=0,0<c<\infty$. By Pro-

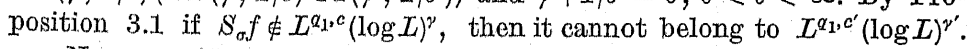

Now consider $a=0, a=1$. Let

$$
f(t)=\left(t^{1 / p_{1}}(1-\ln t)[\ln (1-\ln t)]^{1+s}\right)^{-1} \chi_{(0, A)}(t)
$$

where $A$ and $\varepsilon$ are chosen appropriately small. The function $f$ is positive 
decreasing and belongs to $L^{p_{1}, 1}(\log L)^{0}$. But

$$
S_{\sigma}(f)^{*}(t) \geqslant t^{-1 / q_{1}} \int_{0}^{t^{m}} f(s) s^{1 / p_{1}} \frac{d s}{s} \geqslant c t^{-1 / q_{1}}[\ln (1-\ln t)]^{-s} \chi_{(0, A)}(t) .
$$

But the function on the right, and henee $S_{\sigma}(f)$, does not belong to $L^{q_{1}, c}(\log L)^{\gamma}$. We see that $\varepsilon$ should be chosen less than $1 / c>0$. For $a=0$ and $0<b<1$ we use characteristic functions. As mentioned before, the closed graph theorem implies that (4.1) is equivalent to the statement

$$
\left\|S_{o} f\right\|_{q, c ; \gamma} \leqslant c \mid\left[f \|_{p, a ; \alpha} .\right.
$$

But since $S_{\sigma} \chi_{(0, r)}$ decreases and $S_{\sigma}\left(\chi_{(0, r)}\right)(t) \geqslant \frac{r^{1 / p_{1}}}{t^{1 / \alpha_{1}}} \chi_{\left(r^{1} 1 / m, 1\right)}(t)$,

$$
\left\|S_{\alpha} \chi_{(0, r)}\right\|_{\alpha_{1}, c ; \gamma} \geqslant r^{1 / p_{1}}\left\{\int_{r^{1 / m}}^{1}(1-\ln t)^{\gamma c} \frac{d t}{t}\right\}^{1 / c} \approx r^{1 / 2 \mu_{1}}\left[\ln \left(1-\ln r^{r}\right)\right]^{1 / c} .
$$

Since $\left\|\chi_{(0, r)}\right\|_{p_{1}, a ; 0} \approx r^{1 / p_{1}}$, we see that no relation of type (4.7) can possibly hold.

Proof of 4.2 (b). We first prove sulficiency. Since

$$
\left\|S_{\sigma} f\right\|_{q_{1}, c ; \gamma} \leqslant c\left\|S_{\sigma} f\right\|_{\alpha_{1}, a ; a-1 / a}, \quad \gamma+1 / c=\alpha>0, a \leqslant 0 \leqslant \infty
$$

holds by Theorem 11.2 of [1], and by Proposition 3.1, we see that we only need to establish (4.1) for the case $\gamma=\alpha-1 / a, 0=a$. Since $\left(\mathcal{S}_{\sigma} f\right)^{*}$ $\leqslant S_{\sigma}\left(f_{t^{m}}^{*}\right)$, we consider only positive decreasing $f$. Letiting $g_{1}(t)=$ $t^{-1 / q_{1}} \int_{0}^{t^{m}} f(s) s^{1 / p_{1}} \frac{d s}{s}$ and $g_{2}(t)=S_{\sigma} f(t)-g_{1}(t) \geqslant 0$, we see that it suffices to show

$$
\left\{\int_{0}^{1}\left[t^{1 / \alpha_{1}} g_{i}(t)(1-\ln t)^{\alpha-1 / a}\right]^{a} \frac{d t}{t}\right\}^{1 / a} \leqslant c\|f\|_{p_{1}, a ; \alpha} .
$$

We only estimate this for $g_{1}$ as the case for $g_{2}$ is similar. We let $F(t)$ $=\int_{0}^{t^{m}} f(s) s^{1 / p_{1}} \frac{d s}{s}$ in order to get

$$
\begin{aligned}
& \int_{0}^{1}\left[t^{1 / q_{1}} g_{1}(t)(1-\ln t)^{\alpha-1 / a}\right]^{a} \frac{d t}{t}=\int_{0}^{1} F^{\prime}(t)^{a}(1-\ln t)^{\alpha a-1} \frac{d t}{t} \\
& =c_{x}^{m} \int_{0}^{1} F^{\prime}(t)^{a} d\left[-(1-\ln t)^{a a}\right] \leqslant c \int_{0}^{1}(1-\ln t)^{a a} F^{\prime}(t)^{a-1} f\left(t^{m}\right)\left(t^{m}\right)^{1 / p_{1}-1} d t
\end{aligned}
$$

where we have used integration by parts and the fact that

$$
\varlimsup_{t \rightarrow 0^{+}} F^{\prime}(t)(1-\ln t)^{\alpha} \leqslant \lim _{t \rightarrow 0^{+}}\left\|f \chi_{(0, t)}\right\|_{p_{1}, a ; a}=0 .
$$

But $a-1<0$ and $F(t) \geqslant f\left(t^{m}\right) t^{m / p_{1}}$, so

$$
F(t)^{a-1} \leqslant\left(f\left(t^{m}\right) t^{m / p_{1}}\right)^{a-1} .
$$

Upon making the substitution $u=t^{m}$ and taking $a$ th root of inequality (4.8), we reach the desired conclusion.

For the necessity of $4.2(\mathrm{~b})$ we notice that if $(\gamma, 1 / 0)$ does not belong to the closure of $\boldsymbol{E}(\alpha-1 / a, 1 / a)$ in $\boldsymbol{R}_{+}^{2}$, then $\gamma+1 / 0>\alpha>0$. Consider again the functions $\chi_{(0, r)}$. As before

$$
\left\|S_{\sigma} \chi_{(0, r)}\right\|_{q_{1}, c ; \gamma} \geqslant r^{1 / p_{1}}\left\{\int_{r^{1 / m}}^{1}(1-\ln t)^{\gamma \varepsilon} \frac{d t}{t}\right\}^{1 / c} \approx r^{1 / p_{1}}(1-\ln r)^{\gamma+1 / c}
$$

but $\left\|\chi_{(0, r)}\right\|_{p_{1}, a ; \alpha} \approx r^{1 / p_{1}}(1-\ln r)^{\alpha}$, so no inequality of the form (4.7) can hold as $r \rightarrow 0+$.

Pro of of 4.2 (c). The sufficiency follows from Theorems 11.2 and 12.1 of [1] which say that for $\gamma+1 / c=a+1 / a-1, a \leqslant c \leqslant \infty$, there holds

$$
\left\|S_{\sigma} f\right\|_{q_{1}, c ; \gamma} \leqslant c\left\|S_{\sigma} f\right\|_{q_{1}, a ; \alpha-1} \leqslant c\|f\|_{p_{1}, a ; \alpha} .
$$

For $(\gamma, 1 / c)$ in the interior of $\boldsymbol{E}(\alpha-1,1 / a)$, the embedding Proposition 3.1 applies.

For the necessity we consider only $\gamma+1 / c=a-1+1 / a, 0<c<a$ for the reasons mentioned in part (a), i.e.

We let

$$
\boldsymbol{R}_{+}^{2} \backslash \boldsymbol{E}(\alpha-1,1 / a)=\bigcup_{\substack{\gamma+1 / c=a+1 / a-1 \\ 0<c<<}} \boldsymbol{R}(\gamma, 1 / c) .
$$

$$
f(t)=\left(t^{1 / p_{1}}(1-\ln t)^{\alpha+1 / a}[\ln (1-\ln t)]^{1 / a+\varepsilon}\right)^{-1} \chi_{(0, A)}(t)
$$

where $A$ and $\varepsilon$ are chosen appropriately small. The function $f$ belongs to $L^{p_{1}, a}(\log L)^{a}$, but

$$
\begin{aligned}
S_{\sigma}(f)(t) & \geqslant t^{-1 / q_{1}} \int_{0}^{t^{m}} f(s) s^{1 / p_{1}} \frac{d s}{s} \\
& \geqslant c t^{-1 / q_{1}}\left[(1-\ln t)^{\alpha+1 / a-1}[\ln (1-\ln t)]^{1 / a+s}\right]^{-1} \chi_{(0, A)}(t) .
\end{aligned}
$$

But the function on the right, and hence $S_{\sigma}(f)$, does not belong to $L^{1 / q_{1}, c}(\log L)^{\gamma}$.

\section{References}

[1] C. Bennett and K. Rudnick, On Lorentz-Zygmund spaces, Dissertationes Math. 175, Warszawa 1979, $67 \mathrm{pp}$.

2] A. P. Blozinski, On a convolution theorem for $L(p, q)$ spaces, Trans. Amer. Math. Soc. 164 (1972), pp. 255-265. 
[3] - Convolution of $L(p, q)$ functions, Proc. Amer. Matih. Soc. 32 (1972), pp. 237-240.

[4] D. W. Boyd, The Hilbert transform on rearrangement-invariant spaces, Canad. Math. J. 19 (1967), pp. 599-616.

[5] - Indices of function spaces and their relationship to interpolation, ibid. 21 (1969), pp. 1245-1254.

[6] A. P. Calderón, Spaces between $L^{1}$ and $L^{\infty}$ and the theorem of Marcintiewica, Studia Math. 26 (1966), pp. 273-299.

[7] R. A. DeVore, S. D. Riemenschneider and R. C. Sharpley, Weale interpolation in Banach spaces, J. Functional Analysis 33 (1979), pp. 58-94.

[8] F. Fehér, D. Gaspar and. H. Johnen, Der Konjugiertenoperator auf rearrangement invariant Funktionenräumen, Math. Z. 134 (1973), pp. 129-141.

[9] R. A. Hunt, On $L(p, q)$ spaces, Enseigment Math. 12 (1966), pp. 249-275.

[10] M. Jodeit, Jr. and A. Torchinsky, Inequalities for Fourier transforms, Studia Math. 37 (1971), pp. 245-276.

[11] S. G. Krein and E. M. Semenov, Interpolation of operators of wealkened type, Functional Anal. Appl. 7 (1973), pp. 89-90 (Russian).

[12] G. G. Lor en tz, Bernstein polynomials, University of Toronto Pross, Toronto 1953.

[13]. - On the theory of spaces $A$, Pacific J. Math. 1 (1951), pp. 411-429.

[14] W. A. J. Luxemburg, Banach function spaces, Thesis, Delft Institute of Technology, Assen, Netherlands 1955.

[15] M. Milman and R. C. Sharpley, Convolution operators on $\Lambda_{\alpha}(X)$ spaces, preprint.

[16] R. O'Neil, Convolution operators and $L(p, q)$ spaces, Duke Math. J. 30 (1963), pp. 129-142.

[17] - Fractional integration in Orlicz spaces I, Trans. Amer. Math. Soc. 115 (1965) pp. $300-328$.

[18] R. O'Neil and G. Weiss, The Hilbert transform and rearrangement of functions, Studia Math. 23 (1963), pp. 189-198.

[19] E. M. Semenov, Imbedding theorems for Banach spaces of measurable functions, Soviet Math. Dokl. 5 (1964), pp. 831-834.

[20] R. C. Sharpley, Spaces $\Lambda_{\alpha}(X)$ and interpotation, J. Functional Analysis 11 (1972), pp. 479-513.

[21] - Fractional integration in Orlicz spaces, Proc. Amer. Math. Soc. 59 (1976), pp. 99-106.

[22] - Multilinear weak type interpolation of $m$ n-tuples with applications, Stulia Math. 60 (1977), pp. 179-194.

[23] - Strong interpolation for $\Lambda_{\alpha}(X)$ spaces, preprint.

[24] A. Torchinsky, Interpolation of operations and Orliez classes, Studin Math. 59 (1976), pp. 71-101.

[25] A. Zygmund, Trigonometric series, Cambridge University Press, Cambridge, 1968.

DEPARTMENT OF MATHEMATIOS

UNIVERSITY OF SOUTH CAROLINA

COLUMBIA, SOUTH CAROLINA

Received May 26, 1977

Revised version February 17, 1978
(1318)

\section{Divisible subspaces and problems of automatic continuity*}

by

W. G. BADE (Berkeley, Calif.), P. C. CURTIS, Jr., (Los Angeles, Calif.) and K. B. LAURSEN (Copenhagen)

Abstract. This paper explores the role of divisible subspaces for linear operators in the theory of automatic continuity of homomorphisms between Banach algebras.

§ 0. Introduction. In 1972 Graham Allan [1] showed that the algebra of formal power series could be embedded in a commutative Banach al gebra $B$ if and only if there exists an element $x$ in the radical of $B$ satisfying

$$
\overline{B x^{m+1}} \supseteq B x^{m} \neq 0
$$

for some positive integer $m$. He went on to observe that if this condition is satisfied, then there exists a discontinuous homomorphism $\theta$ from the disc algebra $A(\Delta)$ into the radical of $B$ with unit adjoined which maps the coordinate function $z$ onto $x$. To construct the mapping $\theta$, Allan first shows that if $x$ satisfies the above condition, then there exists a non-zero subspace $Z$ in $\mathrm{rad} B$ such that for every $\lambda \in C$, the complex plane,

$$
(x-\lambda) Z=Z \text {. }
$$

The subspace $Z$, necessarily non-closed, is called $x$-divisible, and the existence of such a subspace is the essential tool in Allan's construction of $\theta$. Indeed in a later paper [29]M. Thomas observes that the existence of an $x$ divisible subspace $Z$ in a radical algebra $R$ is a necessary and sufficient condition for there to exist a discontinuous functional calculus for the element $x$. Since $\sigma(x)=\{0\}$, this is equivalent to the assertion that there exists a discontinuous homomorphism of the disc algebra into the algebra $R$ with unit adjoined which carries $z$ onto $x$

In this paper we shall investigate the relation between the existence of divisible subspaces for elements $x$ in a commutative Banach algebra $B$ and the existence of discontinuous homomorphisms from certain commutative Banach algebras into B. Johnson and Sinclair [20] were the first to investigate the relationship between automatic continuity questions

* This research was supported by the National Science Foundation Grant No. MCS-75-07091 and the Danish Natural Science Research Council. 